hep-th/0410080

SU-ITP-04/38

SLAC-PUB-10790

TIFR/TH/04-27

\title{
A Stringy Cloak for a Classical Singularity
}

\author{
Atish Dabholkar ${ }^{1 \mathrm{a}}$, Renata Kallosh $^{2 \mathrm{~b}}$ and Alexander Maloney ${ }^{2,3 \mathrm{c}}$ \\ ${ }^{1}$ Department of Theoretical Physics, \\ Tata Institute of Fundamental Research \\ Homi Bhabha Road, Mumbai 400005, India \\ 2 Institute for Theoretical Physics, Department of Physics \\ Stanford University, Stanford, CA 94305 \\ 3 Theory Group, Stanford Linear Accelerator Center \\ 2575 Sand Hill Rd, Menlo Park, CA 94025
}

\begin{abstract}
We consider a class of 4D supersymmetric black hole solutions, arising from string theory compactifications, which classically have vanishing horizon area and singular spacetime geometry. String theory motivates the inclusion of higher derivative terms, which convert these singular classical solutions into regular black holes with finite horizon area. In particular, the supersymmetric attractor equations imply that the central charge, which determines the radius of the $A d S_{2} \times S^{2}$ near horizon geometry, acquires a non-vanishing value due to quantum effects. In this case quantum corrections to the Bekenstein-Hawking relation between entropy and area are large. This is the first explicit example where stringy quantum gravity effects replace a classical null singularity by a black hole with finite horizon area.
\end{abstract}

October 2004

\footnotetext{
Work supported in part by the Department of Energy, contract DE-AC02-76SF00515

${ }^{a}$ Email: atish@tifr.res.in

b Email: kallosh@stanford.edu

${ }^{c}$ Email: maloney@slac.stanford.edu
} 


\section{Introduction}

In any quantum theory of gravity, the low-energy effective action is expected to include higher derivative corrections to the Einstein-Hilbert action. In string theory, such corrections can be computed in principle and in many cases may be found explicitly. We will argue that these corrections play an important role in the physics of certain black holes. Many extremally charged black holes and branes have null singularities that are not separated from asymptotic observers by a regular horizon. For a broad class of examples, however, the higher derivative terms completely alter the causal structure of these objects, transforming these apparently singular solutions into regular black holes with finite horizon area. After this 'stringy cloaking', the near horizon geometry becomes $A d S_{2} \times S^{2}$.

We will work in four dimensional, ungauged $N=2$ supergravity [1. In this case the BPS black hole solutions exhibit fixed-point attractor behavior near the horizon, as discovered by Ferrara, Kallosh and Strominger [2, 3. Cardoso, de Wit and Mohaupt described how higher order derivative terms, representing quantum effects, may be added to the action, and found the generalized attractor equations in the presence of these additional terms [4. With Kappeli, they discussed the corresponding black hole solutions [5]. We will follow the work of [4, 5] closely in what follows. Wald has proposed a modification to the usual Bekenstein-Hawking area law [6] in the presence of higher curvature terms [7], which was applied to these black holes by [4]. This work focused primarily on black holes that already have a regular horizon and large area in the leading supergravity approximation, where the entropy receives small but calculable corrections from higher derivative terms. Remarkably, the leading order correction agrees with the counting of black hole microstates performed by Maldacena, Strominger and Witten, and by Vafa [8] Recently, Ooguri, Strominger and Vafa discussed an interesting relation between these black holes and topological string theory [9].

The effects of the higher derivative terms are most dramatic for black holes that classically have zero area and hence zero entropy. It was noticed recently by Dabholkar [10] that for certain two-charge black holes of this type occurring in Type-IIA theory compactified on $\mathbf{K}_{3} \times \mathbf{T}^{2}$, the correction to the entropy coming from higher derivative prepotential-like terms reproduces precisely the logarithm of the number of BPS microstates [11, 12].

Motivated by these results, we examine the space-time geometry of a general class of black holes in $\mathrm{N}=2$ supergravity with higher derivative corrections. In particular, we consider solutions of Type-IIA string theory compactified on a general Calabi-Yau 3-fold, of which the two-charge black hole mentioned above is a special case. We will focus on solutions which classically have null singularities and zero horizon area. We will argue that in these cases the higher curvature terms cause the black holes to develop a regular horizon with non-zero area. ${ }^{\mathrm{d}}$ In the Einstein frame, the radius of the horizon is large for large charges. In addition to making the area non-zero, the higher curvature terms

\footnotetext{
${ }^{\mathrm{d}}$ This result is similar in spirit to the enhancon mechanism of Johnson, Peet and Polchinski 13, although the mechanism is quite different.
} 
significantly modify the Bekenstein-Hawking relation between entropy and area. The net entropy $S$ is then related to the corrected area $A$ by the relation $S=A / 2$, which is distinctly different from the standard relation $S=A / 4$.

The paper is organized as follows. In $\S 2$, we review the basic formalism of the generalized attractor mechanism and the black hole solutions with a general charge configuration for a Calabi-Yau compactification. More details can be found in [14. In $\S 3$ we show that for a large class of charge configurations the classical geometry has a null singularity which is replaced by a smooth horizon once the corrections are taken into account. We discuss some implications when the Calabi-Yau 3-fold is $\mathbf{K}_{3} \times \mathbf{T}^{2}$ in $\S 4$, and summarize our findings in $\S 5$.

\section{Review}

\subsection{Higher Curvature Corrections to $N=2, D=4$ Supergravity}

Our starting point is the $N=2$ superconformal theory of gravity in four dimensions described by [1, which is subsequently gauge fixed to give $N=2$ Poincaré supergravity. The field content is a Weyl multiplet, which contains the graviton as well as other physical and auxiliary fields, along with $n+1$ vector multiplets. One of these vector fields is the graviphoton, which is not contained in the Weyl multiplet. One can define a superfield $\mathbf{W}_{a b}^{i j}$, where $i, j=1,2$ are spinor indices and $a, b$ are local Lorentz indices, which contains the field strengths of the Weyl multiplet as well as an anti-selfdual auxiliary field $T^{a b i j}$. The lowest component of the associated chiral multiplet $\mathbf{W}^{2}=\left(\mathbf{W}^{a b i j} \varepsilon_{i j}\right)^{2}$ is $\hat{A}=\left(T^{a b i j} \varepsilon_{i j}\right)^{2}$. The couplings between the vector multiplets and the Weyl multiplet are summarized by a holomorphic function

$$
F\left(X^{I}, \hat{A}\right), \quad I=0,1, \ldots, n
$$

which is homogeneous of degree two. When $F$ is independent of $\hat{A}$, this is the usual holomorphic prepotential, which depends on the scalar components $X^{I}$ of the $n+1$ vector multiplets.

The supergravity field equations are covariant under symplectic $\operatorname{SP}(2 n+2 ; \mathbf{Z})$ transformations, which are a generalization of electric-magnetic duality. These transformations act linearly on the two $(2 n+2)$-component vectors

$$
V=\left(\begin{array}{c}
X^{I} \\
F_{I}(X, \hat{A})
\end{array}\right) \quad \text { and } \quad\left(\begin{array}{c}
p^{I} \\
q_{I}
\end{array}\right) .
$$

Here $F_{I}=\partial F / \partial X^{I}$, and $p^{I}, q_{I}$ are the electric and magnetic charges of a given solution. We can use these vectors to define the central charge

$$
Z=e^{\mathcal{K} / 2}\left(p^{I} F_{I}(X, \hat{A})-q_{I} X^{I}\right),
$$


where $e^{-\mathcal{K}}=i\left[\bar{X}^{I} F_{I}(X, \hat{A})-\bar{F}_{I}(\bar{X}, \overline{\hat{A}}) X^{I}\right]$, which is invariant under symplectic transformations.

When $F$ depends on $\hat{A}$ the action contains higher curvature couplings between the Weyl and vector multiplets. The bosonic Lagrangian contains the terms

$$
8 \pi \mathcal{L}=-\frac{1}{2} e^{-\mathcal{K}} R+\frac{1}{2}\left(i F_{\hat{A}} \hat{C}+\text { h.c. }\right)+\cdots,
$$

where $\hat{C}=64 C^{-\mu \nu \rho \sigma} C_{\mu \nu \rho \sigma}^{-}+16 \varepsilon_{i j} T^{\mu \nu i j} f_{\mu}{ }^{\rho} T_{\rho \nu k l} \varepsilon^{k l}+\cdots$. Here $C_{\mu \nu \rho \sigma}^{-}$denotes the antiselfdual part of the Weyl tensor $C_{\mu \nu \rho \sigma}$, and $f_{\mu}{ }^{\nu}=\frac{1}{2} R_{\mu}{ }^{\nu}-\frac{1}{12} R \delta_{\mu}{ }^{\nu}+\cdots$. To fix the local scale invariance of the theory and obtain $N=2$ Poincaré supergravity, we must set $e^{-\mathcal{K}}$ to one. The first term in the action is then the properly normalized Einstein-Hilbert term.

\subsection{Black Hole Solutions}

Black hole solutions to this theory may be parameterized in terms of two functions $f(r)$ and $g(r)$ as

$$
d s^{2}=-e^{2 g(r)} d t^{2}+e^{2 f(r)}\left[d r^{2}+r^{2}\left(\sin ^{2} \theta d \phi^{2}+d \theta^{2}\right)\right] .
$$

At $r \rightarrow \infty, e^{2 g(r)} \rightarrow 1$ and $e^{2 f(r)} \rightarrow 1$, so the metric is asymptotically flat. As $r \rightarrow 0$, it was shown by 4 , that

$$
e^{2 f(r)}=e^{-2 g(r)}=\frac{|Z \bar{Z}|_{r=0}}{r^{2}}
$$

where $Z$ is the central charge defined above. When $\left.Z\right|_{r=0}$ is non-vanishing the solution becomes $A d S_{2} \times S_{2}$ at small $r$, and the black hole has a finite horizon area

$$
A=4 \pi|Z \bar{Z}|_{r=0}
$$

However, when $|Z \bar{Z}|_{r=0}=0$ the solution has a null singularity: typically $e^{2 f(r)}=e^{-2 g(r)} \sim$ $1 / r$, and the horizon at $r=0$ is coincident with the null singularity.

Equation (7) may also be derived by imposing unbroken $\mathrm{N}=2$ supersymmetry at the horizon. Starting from the gravitino variation

$$
\delta \psi_{\mu}^{i}=2 \mathcal{D}_{\mu} \epsilon^{i}-\frac{1}{8} T^{a b i j} \gamma_{a b} \gamma_{\mu} \epsilon_{j}
$$

the gauge invariant condition $\delta \mathcal{D}_{[\mu} \psi_{\nu]}^{i}=0$ implies a relation between the curvature of a near horizon $A d S_{2} \times S^{2}$ and the value of the central charge $Z$ at $r=0$. This procedure is explained in detail in [15].

To evaluate $\left.Z\right|_{r=0}$, we may use the generalized attractor equations [4

$$
\bar{Z}\left(\begin{array}{c}
X^{I} \\
F_{I}(X, \hat{A})
\end{array}\right)-Z\left(\begin{array}{c}
\bar{X}^{I} \\
\bar{F}_{I}(\bar{X}, \overline{\hat{A}})
\end{array}\right)=i e^{-\mathcal{K} / 2}\left(\begin{array}{c}
p^{I} \\
q_{I}
\end{array}\right)
$$


which follow from the constraint of unbroken $\mathrm{N}=2$ supersymmetry at the horizon. When there are no quantum corrections, i. e. when the prepotential $F$ is independent of $\hat{A}$, these are the usual attractor equations 3. When $F$ depends on $\hat{A}$, consistency of the construction requires that $\hat{A}=-64 e^{-\mathcal{K}} \overline{\bar{Z}}^{-2}$.

The authors of [4] considered regular black hole solutions which classically have nonzero horizon area. In the presence of higher curvature corrections, the entropy is not given by the usual Bekenstein-Hawking formula. Instead, following Wald's proposal [7, the authors of [4] found

$$
S=\pi\left[|Z|^{2}-256 \operatorname{Im}\left[F_{\hat{A}}\left(X^{I}, \hat{A}\right)\right]\right], \quad \text { with } \quad \hat{A}=-64 \bar{Z}^{-2} \mathrm{e}^{-\mathcal{K}}
$$

where $X^{I}$ and $Z$ are fixed by the stabilization equations at $r=0$. The first term is one quarter the horizon area (including higher derivative corrections), while the second term is the novel contribution to the entropy due to Wald.

Following [16], we will introduce the rescaled variables $Y^{I}=\mathrm{e}^{\mathcal{K} / 2} \bar{Z} X^{I}$ and $\Upsilon=\mathrm{e}^{\mathcal{K}} \bar{Z}^{2} \hat{A}$. Using the homogeneity property of $F$, we find

$$
Z \bar{Z}=p^{I} F_{I}(Y, \Upsilon)-q_{I} Y^{I}
$$

The stabilization equations (9) become

$$
\begin{gathered}
Y^{I}-\bar{Y}^{I}=i p^{I} \\
F_{I}(Y, \Upsilon)-\bar{F}_{I}(\bar{Y}, \bar{\Upsilon})=i q_{I} .
\end{gathered}
$$

The entropy formula (10) takes the form $S=\pi\left[Z \bar{Z}-256 \operatorname{Im} F_{\Upsilon}(Y, \Upsilon)\right]$ with $\Upsilon=-64$.

\subsection{Calabi-Yau Black Holes}

In this paper, we will focus on black holes found by compactifying type-IIA string theory on a large Calabi-Yau threefold. The prepotential has zero and linear order dependence on $\hat{A}$, so that

$$
F(Y, \Upsilon)=\frac{D_{A B C} Y^{A} Y^{B} Y^{C}}{Y^{0}}+d_{A} \frac{Y^{A}}{Y^{0}} \Upsilon
$$

Here $I=0, \ldots, n, A=1, \ldots, n$ and

$$
D_{A B C}=-\frac{1}{6} C_{A B C}, d_{A}=-\frac{1}{24} \frac{1}{64} c_{2 A}
$$

depend on the Calabi-Yau four-cycle intersection numbers $C_{A B C}$ and second Chern-class coefficients $c_{2 A}$ [17]. The classical solution to the attractor equations, where $\Upsilon \rightarrow 0$ in (14), was found by Shmakova [18]. 
The quantum corrected solution, including the second term in (14), is somewhat more complicated. In this case the Lagrangian (44) contains a higher curvature term proportional to $c_{2 A} z^{A} R^{2}$, where $z^{A}=Y^{A} / Y^{0}$. Let us take $p^{0}=0$ and assume that there is a solution with $Y^{0} \neq 0$. The solution to the generalized attractor equation was found in this case to be 4 ]

$$
Y^{0}=\bar{Y}^{0}, \quad\left(Y^{0}\right)^{2}=\frac{D-4 d_{A} p^{A} \Upsilon}{4 \hat{q}_{0}}, \quad Y^{A}=\frac{1}{6} Y^{0} D^{A B} q_{B}+\frac{1}{2} i p^{A}
$$

Here, following [18, we denote $D \equiv D_{A B C} p^{A} p^{B} p^{C}, D_{A B} \equiv D_{A B C} p^{C}$, and define $D^{A B}$ by $D_{A B} D^{B C} \equiv \delta_{A}^{C}$. We have also introduced $\hat{q}_{0}=q_{0}+\frac{1}{12} D^{A B} q_{A} q_{B}$, which we will take to be negative. Note that this formula is valid only when the matrix $D_{A B}=D_{A B C} p^{C}$ is invertible. In the next section we will consider general configurations where this may not be the case. We must also take $p^{A}>0$ to keep $\left(Y^{0}\right)$ real.

Putting this together, we have the corrected formula for the horizon area

$$
A=4 \pi|Z|_{r=0}^{2}=16 \pi Y^{0}\left|\hat{q}_{0}\right|-8 \pi \frac{d_{A} p^{A}}{Y^{0}} \Upsilon .
$$

The corrected entropy formula, using $\Upsilon=-64$, is

$$
S=4 \pi Y^{0}\left|\hat{q}_{0}\right|
$$

This agrees with the microscopic value computed in 8 .

\section{$3 \quad$ A Stringy Cloak for a Null Singularity}

In this section we study supersymmetric solutions with vanishing classical horizon area, and conclude that quantum corrections due to $R^{2}$-terms lead to non-zero area.

Consider a Calabi-Yau black hole with vanishing graviphoton magnetic charge $p^{0}=0$. In the absence of higher curvature terms, i.e. taking $\Upsilon \rightarrow 0$, the area and entropy take the classical values

$$
S_{c l}=\frac{A_{c l}}{4}=4 \pi Y_{c l}^{0}\left|\hat{q}_{0}\right|=2 \pi \sqrt{\hat{q}_{0} D_{A B C} p^{A} p^{B} p^{C}}
$$

We have used here the attractor equations to fix the classical value of $Y^{0}$ at $r=0$,

$$
\left(Y^{0}\right)_{c l}^{2}=\frac{D}{4 \hat{q}_{0}}
$$

We will consider black holes with charges $p^{A}$ such that

$$
D \equiv D_{A B C} p^{A} p^{B} p^{C}=0
$$


vanishes. We see from (19) that the classical value for the area vanishes. Note that the classical value of $Y^{0}$ given by (20) vanishes, so the attractor solutions presented above become singular.

Fortunately, quantum corrections to the prepotential allow one to consistently solve the attractor equations. Making the ansatz

$$
Y^{A}=w^{A}+\frac{i}{2} p^{A}
$$

where the $w^{A}$ are real, the attractor equations become

$$
D_{A B} w^{B}=\frac{1}{6} Y^{0} q_{A}, \quad D_{A B} w^{A} w^{B}=-\frac{1}{3}\left(q_{0}\left(Y^{0}\right)^{2}+d_{A} p^{A} \Upsilon\right) .
$$

When $D_{A B}$ is invertible, the solution is given by (16),

$$
Y^{0}=\sqrt{\frac{c_{2 A} p^{A}}{24\left|\hat{q}_{0}\right|}} .
$$

The quantum corrected horizon area becomes

$$
A=4 \pi|Z|_{r=0}^{2}=8 \pi \sqrt{\frac{c_{2 A} p^{A}\left|\hat{q}_{0}\right|}{24}} .
$$

This is non-zero provided $c_{2 A} p^{A}$ and $\hat{q}_{0}$ are non-vanishing. Note that $A$ depends on the topology of the Calabi-Yau (via the Chern classes $c_{2 A}$ ) as well as on the charges of the black hole. The area is large when $\hat{q}_{0}, c_{2 A} p^{A}$ are large. Formula (25) may change once higher order $\alpha^{\prime}$ and $g_{s}$ corrections are included.

If the matrix $D_{A B}$ is not invertible, then the solution is a little more complicated. In this case, as may be seen from (23), one must impose additional conditions on the electric charges $q_{A}$ to keep $Y^{0}$ non-zero. The simplest constraint is $q_{A}=0$, in which case the above solution is correct provided we set $\hat{q}_{0}=q_{0}$. More generally, the charge vector $q_{A}$ must be orthogonal to the kernel of the matrix $D_{A B}$. In this case we have $\hat{q}=q_{0}+\frac{1}{12} \hat{D}^{A B} q_{A} q_{B}$, where $\hat{D}^{A B}$ is the inverse of $D_{A B}$ in the subspace orthogonal to it's kernel.

For all of the configurations described above, the corrected entropy is

$$
S=4 \pi Y^{0}\left|\hat{q}_{0}\right|=4 \pi \sqrt{\frac{c_{2 A} p^{A}\left|\hat{q}_{0}\right|}{24}} .
$$

The quantum corrected entropy-area relation for this class of black holes becomes

$$
S=\frac{A}{2} \text {. }
$$

We should emphasize that we have derived this relation only for this specific class of solutions, where supersymmetry and symplectic invariance strongly constrain the result. In particular, both $S$ and $A$ are symplectic invariants, so it is not surprising that they remain proportional even after higher order corrections are included. It would be interesting to find the analogous formula for other types of black holes. 


\section{Two-charge Black Holes in $K_{3} \times T_{2}$ Compactification}

In this section we specialize to Type-IIA string theory compactified on $\mathbf{K}_{3} \times \mathbf{T}_{2}$ considered in [10. There are 23 two-cycles, provided by the torus $T_{2}$ and the 22 two-cycles of $K_{3}$. The non-vanishing intersection numbers are

$$
C_{1 a b}=C_{a b},
$$

where $a=2, \ldots, 23$ runs over the two-cycles of $K_{3}$ and $C_{a b}$ is the intersection matrix on $K_{3}$. The second Chern class is $c_{2,1}=24$.

One virtue of this special example is that the power series expansion of the holomorphic function $F\left(X^{I}, \hat{A}\right)$ terminates at first order in $\hat{A}$. Moreover, the full quantum corrected expression for $F$ can be evaluated exactly (see e.g. [10]). This exact expression for $F$ includes the terms listed in equation (14), as well as corrections that are suppressed when the volume of $K_{3} \times T_{2}$ is large. This allows a more detailed comparison of the microscopic degeneracy with the black hole partition function defined by [9] to all orders in the inverse charge in an asymptotic expansion.

We consider the configuration where $q_{0}=n$ and $p^{1}=w$ are non-zero and the rest of the charges vanish. This corresponds to a configuration of $w$ D4-branes wrapping the $\mathbf{K}_{3}$ with $n$ D0-branes sprinkled on the worldvolume. This state is dual to a perturbative state with winding number $w$ and quantized momentum $n$ in the heterotic theory compactified on $\mathbf{T}^{4} \times \mathbf{T}^{2}$. The condition (21) is satisfied for this configuration, so the black holes classically have zero area. The quantum corrected area is

$$
A=8 \pi \sqrt{p^{1}\left|q_{0}\right|}
$$

and the corrected entropy is

$$
S=4 \pi \sqrt{p^{1}\left|q_{0}\right|}=\frac{A}{2} .
$$

The corrected geometry of the black hole near the horizon is given by $A d S_{2} \times S^{2}$. In Einstin frame, the radius of the sphere goes as $N^{\frac{1}{4}}$ where $N \equiv|n w|$ can be taken to be large. The attractor equations also determine the value of the heterotic dilaton $\phi$ at the horizon. Recall that $e^{-2 \phi}$ is given by the imaginary part of $\frac{Y^{1}}{Y^{0}}$ which gives $e^{-2 \phi} \sim \sqrt{N}$ from the solution of the attractor equations for our charge configuration [10. The closed-string loop-counting parameter $g_{s}^{2}=e^{2 \phi}$ thus varies in the black hole geometry and reaches a constant value at the horizon that goes as $\frac{1}{\sqrt{N}}$ and is thus vanishingly small for large N.

Given this value of the dilaton, the radius of curvature at the horizon is of the order of string scale in the string metric even though it is large in the Einstein metric. This is consistent with the heuristic idea of a string-scale 'stretched horizon' 19 for the zero area extremal black holes [20] in the heterotic description. Note that while the string metric 
determines the sigma-model couplings, the Einstein metric is the one that is invariant under duality.

In the Type-IIB description, this system is dual to the D1-D5 system where the geometry is known to be desingularized already at the classical level without the inclusion of higher curvature terms [21, 22. It was found that there are many such smooth geometries, which are dual to the oscillating string solutions of [23, 24] that are specified by an arbitrary profile of the left-moving oscillations. The multiplicity of these smooth solutions then accounts for the degeneracy of the corresponding BPS states. We are finding here instead that the inclusion of higher curvature terms generates a regular horizon and the entropy associated with this horizon accounts for the degeneracy of these states. It would be interesting to understand in detail how these two dual pictures are compatible with each other.

The entropy of these black holes no longer satisfies the Bekenstein-Hawking area formula but also includes the correction due to higher derivative terms given by Wald's formula. For the class of solutions considered here, we find that the entropy is given by $S=A / 2 \equiv A / 4+A / 4$. Thus the correction to the entropy is of the same order as the Bekenstein-Hawking entropy. Note that $A$ here is the quantum corrected area and is quite different from the classical area which vanishes.

This raises an important physical question. The Bekenstein-Hawking formula for the entropy allows one to view the area theorems of black hole dynamics in classical general relativity as special cases of black hole thermodynamics. In particular, the fact that the area of black holes always increases in physical processes can then be viewed as a special case of the second law of thermodynamics that the entropy always increases in physical processes. For our black holes, since the entropy is quite different from classical area, it is important to show that the geometric quantity defined as the entropy by Wald's formula always increases in a physical process. This is necessary in order that the second law of thermodynamics is obeyed with the definition of entropy by Wald which appears in the first law of black hole thermodynamics. Note that the entropy thus defined is in perfect agreement with the counting of BPS microstates and the specific corrections to the Einstein-Hilbert term used here are well-motivated by string theory. Therefore, this particular action and slightly off extremal black holes in this system could be a good starting point for considering possible generalizations of area theorems of classical general relativity within string theory.

\section{Conclusions}

In conclusion, we have found a large family of classically singular, zero area solutions which are converted into regular black holes by higher derivative corrections to the supergravity action. This class includes the two-charge solutions of string theory on $K_{3} \times T_{2}$ studied recently in [10]. 
The entropy-area relation for this particular class of black holes is $S=\frac{A}{2}$, which differs from the well known classical relation $S=\frac{A}{4}$. However, we expect that this modified version the Bekenstein-Hawking formula applies only to this special class of Calabi-Yau black holes.

It would be interesting to investigate other black hole solutions with vanishing classical horizon area. We would also like to understand any relation between our stringy cloaking device and the proposal of [9], as well as the effects of higher order $\alpha^{\prime}$ and $g_{s}$ corrections on our results. We leave this for future work.

\section{Acknowledgements}

We would like to thank J. Harvey, G. Horowitz, J. Hsu, S. Kachru, A. Linde, E. Martinec, S. Mathur, J. McGreevy, M. Shmakova, E. Silverstein, S. Shenker, L. Susskind, R. Wald, and M. Zagermann for helpful discussions. This work was supported by NSF grant 0244728, and by the Department of Energy under contract number DE-AC02$76 \mathrm{SF} 00515$.

\section{References}

[1] B. de Wit, J. W. van Holten and A. Van Proeyen, Nucl. Phys. B167 (1980) 186, Nucl. Phys. B184 (1981) 77; B. de Wit and A. Van Proeyen, Nucl. Phys. B245 (1984) 89; B. de Wit, P. G. Lauwers and A. Van Proeyen, Nucl. Phys. B255 (1985) 569.

[2] S. Ferrara, R. Kallosh and A. Strominger, Phys. Rev. D52 (1995) 5412, hep-th/9508072; A. Strominger, Phys. Lett. B383 (1996) 39, hep-th/9602111.

[3] S. Ferrara and R. Kallosh, Phys. Rev. D54 (1996) 1514, hep-th/9602136 Phys. Rev. D54 (1996) 1525, hep-th/9603090.

[4] G. Lopes Cardoso, B. de Wit and T. Mohaupt, Phys. Lett. B 451, 309 (1999) [arXiv hep-th/9812082; G. Lopes Cardoso, B. de Wit and T. Mohaupt, Fortsch. Phys. 48, 49 (2000) [arXiv:hep-th/9904005.

[5] G. L. Cardoso, B. de Wit, J. Kappeli and T. Mohaupt, Fortsch. Phys. 49, 557 (2001) [arXiv hep-th/0012232 . G. L. Cardoso, B. de Wit, J. Kappeli and T. Mohaupt, arXiv:hep-th/0003157. G. Lopes Cardoso, B. de Wit, J. Kappeli and T. Mohaupt, JHEP 0012, 019 (2000) [arXiv:hep-th/0009234].

[6] S. W. Hawking, Phys. Rev. Lett. 26 (1971) 1344; J. M. Bardeen, B. Carter and S. W. Hawking, Commun. Math. Phys. 31 (1973) 161; J. D. Bekenstein, Phys. Rev. D7 (1973) 2333; Phys. Rev. D9 (1974) 3292; S. W. Hawking, Comm. Math. Phys. 43 (1975) 199. 
[7] R. M. Wald, Phys. Rev. D48 (1993) 3427, gr-qc/9307038, T. Jacobson, G. Kang and R. C. Myers, Phys. Rev. D49 (1994) 6587, gr-qc/9312023, gr-qc/9502009, V. Iyer and R. M. Wald, Phys. Rev. D50 (1994) 846, gr-qc/9403028, R. M. Wald, gr-qc/9702022; gr-qc/9901033.

[8] J. M. Maldacena, A. Strominger and E. Witten, J. High Energy Phys. 12 (1997) 2, hep-th/9711053; C. Vafa, Adv. Theor. Math. Phys. 2 (1998) 207, hep-th/9711067.

[9] H. Ooguri, A. Strominger and C. Vafa, arXiv:hep-th/0405146.

[10] A. Dabholkar, arXiv:hep-th/0409148.

[11] A. Dabholkar and J. A. Harvey, Phys. Rev. Lett. 63, 478 (1989).

[12] A. Dabholkar, G. W. Gibbons, J. A. Harvey and F. Ruiz Ruiz, Nucl. Phys. B 340, 33 (1990).

[13] C. V. Johnson, A. W. Peet and J. Polchinski, Phys. Rev. D 61, 086001 (2000) [arXiv hep-th/9911161.

[14] T. Mohaupt, Fortsch. Phys. 49, 3 (2001) [arXiv:hep-th/0007195].

[15] A. H. Chamseddine, S. Ferrara, G. W. Gibbons and R. Kallosh, Phys. Rev. D 55, 3647 (1997) [arXiv:hep-th/9610155].

[16] K. Behrndt, G. L. Cardoso, B. de Wit, R. Kallosh, D. Lüst and T. Mohaupt, NP488 (1997) 236, hep-th/9610105

[17] M. Bershadsky, S. Cecotti, H. Ooguri and C. Vafa, Nucl. Phys. B405 (1993) 279, hep-th/9302103.

[18] M. Shmakova, Phys. Rev. D56 (1997) 540, hep-th/9612076

[19] L. Susskind, Phys. Rev. D 49, 6606 (1994) [arXiv hep-th/9308139.

[20] A. Sen, Nucl. Phys. B 440, 421 (1995) [arXiv hep-th/9411187]. A. Sen, Mod. Phys. Lett. A 10, 2081 (1995) [arXiv:hep-th/9504147.

[21] O. Lunin and S. D. Mathur, Nucl. Phys. B 623, 342 (2002) [arXiv hep-th/0109154.

[22] O. Lunin, J. Maldacena and L. Maoz, arXiv hep-th/0212210.

[23] C. G. . Callan, J. M. Maldacena and A. W. Peet, Nucl. Phys. B 475, 645 (1996) [arXiv:hep-th/9510134.

[24] A. Dabholkar, J. P. Gauntlett, J. A. Harvey and D. Waldram, Nucl. Phys. B 474, 85 (1996) [arXiv:hep-th/9511053. 